\title{
Investigation of THDOC effects on pathophysiological signs of Alzheimer's disease as an endogenous neurosteroid: inhibition of acetylcholinesterase and plaque deposition
}

\author{
Saleh $\mathrm{H}^{1}$, Sadeghi $\mathrm{L}^{2}$ \\ Department of Physiology, Payam Noor University of Iran, Iran. l.sadeghi@tabrizu.ac.ir
}

\begin{abstract}
Alzheimer's disease (AD) is an advanced neurodegenerative disorder greatly accompanied by acetylcholinesterase (AChE) activation and amyloid plaque deposition. Tetrahydrodeoxycorticosterone (THDOC) is an endogenous neurosteroid that is reduced in $A D$ patient according to previous results. It has neuroprotective effects and plays important role in neurological diseases. By considering AChE role in AD, this study investigated THDOC effects on catalytic and non-catalytic functions of the enzyme. Inhibitory effect of THDOC on hydrolytic activity of AChE was confirmed by in vitro assay $\left(\mathrm{IC}_{50}=5.68 \mu \mathrm{M}\right)$. Molecular docking analysis revealed THDOC bound tightly to the catalytic site of enzyme and inhibited substrate binding. According to in vivo experiments, neurosteroid administration causes inhibition of hyper-activated AChE in hippocampus related to rat model of AD. Staining of hippocampus tissue by plaque specific dye approved THDOC reduced plaque numbers and size in $A D$ rats. Histological and immunoblotting experiments showed neurosteroid administration improved neurodegeneration and neuronal damages in AD rats that lead to improved spatial learning ability. Overall this study suggests, THDOC is an endogenous regulator for AChE. By considering pathophysiological and molecular similarities between $A D$ and animal model, our results highlight THDOC as a potential therapeutic strategy in patients suffering from AD or similar cognitive disorders (Fig. 6, Ref. 28). Text in PDF www.elis.sk.

KEY WORDS: tetrahydrodeoxycorticosterone, acetylcholinesterase, non-catalytic function, amyloid plaque deposition, nucleus basalis of Meynert lesioned rats, neurodegeneration.
\end{abstract}

\section{Introduction}

Alzheimer's disease (AD) is a progressive dementia that is characterized by memory loss and cognitive dysfunction (1). Hallmarks of AD are amyloid plaque deposition and acetylcholine $(\mathrm{ACh})$ reduction as a result of acetylcholinesterase (AChE) hyper-activation $(2,3)$. Cumulative evidences suggest AChE plays important role in incidence and progress of $\mathrm{AD}$ in patients and animal models (2). Therefore AChE inhibitors are considered as an effective group of medications in AD therapy such as donepezil, galantamine, and rivastigmine (2). AChE which is a member of $\alpha / \beta$-hydrolase fold family could degrade ACh in the synaptic cleft very fast and attenuates cholinergic impulse in nerves (4). This multifunctional enzyme contributes in multiple biological processes in addition to catalytic activity such as cell differentiation, dendrite and axon formation, cell adhesion and neuro-

\footnotetext{
${ }^{1}$ Department of Physiology, Payam Noor University of Iran, Iran, and ${ }^{2}$ Department of Animal Biology, Faculty of Natural Science, University of Tabriz, Tabriz, Iran
}

Address for correspondence: L. Sadeghi, Subdivision of Biochemistry, Department of Animal Biology, Faculty of Natural Science, University of Tabriz, Tabriz, Iran, P.O. Box 5166616471, Tabriz, Iran.

Phone: +9841.33392688, Fax: +9841.33356027 degeneration in central nervous system (CNS) (5). Previously approved $\mathrm{AChE}$ plays undeniable role in $\mathrm{A} \beta$ plaque deposition as a molecular chaperon (3). Structure of this protein has two ligand interacting sites that mediate catalytic and non-catalytic functions: an active (hydrolytic) site at the bottom of a deep and narrow gorge contains catalytic residues and a peripheral anionic site (PAS) that consists of negatively charged and hydrophobic residues surrounding the entrance of the gorge $(2,6)$. Both of the sites could interact with natural and synthetic small compounds by mediating aromatic and hydrophobic residues (2). Previous results confirmed neurosteroids affect ACh content in CNS by unknown mechanisms (7) that could be done by mediating AChE enzyme. Neurosteroids are steroid molecules that are synthesized or metabolized within the brain and influence the development and function of the CNS and modulate neuronal excitability and viability (8). Some steroid hormones that reach to the brain by circulation serve as precursors for the synthesis of secondary neurosteroids, but strong evidences confirmed local and de novo production of neurosteroids in the hippocampus and other brain structures (9). Tetrahydrodeoxycorticosterone (THDOC) is an endogenous neurosteroid synthesized from deoxycorticosterone by the action of two enzymes, $5 \alpha$-reductase and $3 \alpha$-hydroxysteroid dehydrogenase, and is reduced in some neurological disease such as epilepsy and depression (10). THDOC is a potent positive allosteric modu- 
lator of the GABA receptor, and has sedative, anxiolytic and anticonvulsant effects (11). Previous studies showed THDOC could pass through blood brain barrier $(\mathrm{BBB})$ and reach to the neurons and affect inflammation, excitation and apoptosis (12), therefore possibly improve neurodegeneration in Parkinson and Alzheimer disease also. This study aimed to investigate THDOC effects on AChE activity. The best binding site of goal neurosteroid on AChE were investigated by molecular docking analysis. Finally potential of THDOC to improve pathophysiological sings of AD was evaluated by behavioral analysis, immunoblotting assessment and histological studies on Nucleus basalis of Meynert lesioned (NBML) rats as approved animal model of AD (13). As previous results have shown, hippocampal tissue plays an important role in hippocampal-dependent learning, memory and cognitive disorders and also is a good source of AChE (14), so we used hippocampal tissue as target in all of the experiments.

\section{Material and methods}

\section{Chemicals}

Tetrahydrodeoxycorticosterone was obtained from SigmaAldrich, USA. Anti-beta amyloid 1-42 antibody (ab10148), rat specific anti-amyloid precursor protein (APP) antibody (ab2072) prepared from Abcam Company. Rat specific monoclonal AntiGlial Fibrillary Acidic Protein (GFAP) antibody (G3893) purchased from Sigma Aldrich Company.

\section{Neurostroid effects on hippocampal AChE activity}

We used adult rat hippocampus homogenate in cold buffer phosphate, $28 \mathrm{mM}$ (pH 7.8) containing Triton X100 as AChE source (15). Enzyme assay was done according to previously established method (15), which is based on the formation of yellow 5-thio-2-nitrobenzoate anion produced in the reaction between 5,5'-Dithiobis (2-nitrobenzoic acid) (DTNB) and thiocholine that was produced by $\mathrm{AChE}$ by hydrolyzing $\mathrm{ACh}$ as substrate. The final concentrations of DTNB and substrate were $0.33 \mathrm{mM}$ and 1.56 $\mathrm{mM}$, respectively, in the assay medium. Absorbance of the solution was measured at $430 \mathrm{~nm}$ using a spectrophotometer. The activity was calculated as nanomole of ATCh hydrolyzed per minute per milligram protein in the presence and absence of THDOC. Protein concentration was evaluated according to Bradford method in the homogenate (16).

\section{Molecular docking analysis}

Auto Dock software (version 4.2) was used to simulate the binding process of THDOC molecules to AChE protein in molecular docking method (17). The crystal structure of AChE (PDB ID: 1DX6) was obtained from the Protein Data Bank (https://www. rcsb.org/). Since different subunits of the protein are completely similar to each other and have same structure, the chain A was selected to perform docking analysis accurately. Polar hydrogen atoms were added into the target protein (AChE) and Kollman charges were calculated. The 3D molecular structure of THDOC was obtained from the PubChem website (https://pubchem.ncbi. nlm.nih.gov/compound/101771) (PubChem CID for THDOC:
CID 101771). The structural conformation was minimized by Gaussian 03 programs based on theoretical level of B3LYP with 6-31 $\mathrm{G}$ basis set. In addition, the rotation of ligand molecule was defined and rotatable bonds were detected. Lamarckian genetic algorithm (LGA) search method was used for docking calculation to find the best binding site of THDOC on AChE protein. All other calculation parameters were set on default. Chimera 1.10.1 program was used to visualize the obtained THDOC-AChE complex structure (18).

\section{In vivo experimental design}

In vivo study was conducted on experimental animals using 4.5-6 months adult male Wistar rats weighing 250-300 g obtained from the animal house. Testing was carried out at $20-25^{\circ} \mathrm{C}(12$ hours light and 12 hours dark). Municipal tap water was used as drinking water and compressed food as nutrition. NBM lesioned rats were prepared as AD model as follow: Rats were anesthetized with ketamine (125 mg/kg, i.p) and xylazine (10 mg/kg, i.p) and then placed in a Stoelting stereotaxic apparatus. The midline of clean scalp was notched and a burr hole was drilled through the skull and ibotenic acid was injected at NBM nucleus with coordination as follow: $\mathrm{AP}=1.2, \mathrm{ML}= \pm 3.2, \mathrm{DV}=7.5 \mathrm{~mm}$ from surface skull (19). Each side of NBM was injected by $1 \mu \mathrm{l}$ of $5 \mu \mathrm{g} / \mu \mathrm{l}$ ibotenic acid solution with microinjection pump at the speed of $120 \mu \mathrm{l} / \mathrm{h}$. After recovery (7 days) animals were divided randomly into four groups as follows:

1) Control: rats were submitted to surgery and injected by 1 $\mu \mathrm{l}$ saline (as equivalent shock obtained by surgery and local injection) and were orally administrated by saline after recovery during 25 days.

2) Rat model of AD: NBM lesioned rats (NBML) in which both NBM nucleus were bilaterally destroyed by ibotenic acid during surgery. These rats received saline orally after recovery for 25 days.

3) AD rats treated by neurosteroid: NBM nuclei were bilaterally destroyed by ibotenic acid and after recovery $\mathrm{AD}$ rats were treated orally by $30 \mathrm{mg} / \mathrm{kg}$ THDOC during 25 days. Dose of THDOC and experimental time duration were selected according to previous studies and in vitro enzyme assay results (20).

\section{Behavioral assessment by Morris water maze}

The Morris water maze (MWM) is a spatial learning test for rodents; navigation from start locations around the perimeter of an open swimming field to an escape platform according to distal marks. The swim maze is a circular bath (diameter $140 \mathrm{~cm}$ ) and its temperature controlled at $25{ }^{\circ} \mathrm{C}$, the apparatus is similar to that described by Morris (21). The test room was ventilated at a constant temperature of $25^{\circ} \mathrm{C}$. A platform was placed at a constant position within the bath at $1 \mathrm{~cm}$ below the water level (30 $\mathrm{cm}$ depth). Before testing, all the rats were trained for $60 \mathrm{~s}$ without the platform. Each rat passed one test session on each of 3 following days after experimental duration. After training, rats were presented 5 tests in each three consecutive days. During the test, the rat was located in the water at the same point in the pool and allowed to swim around to reach to the platform and escape from 


\section{8-154}

the water. The rat was allowed to remain on the platform for $30 \mathrm{~s}$ before the next test. If a rat failed to reach the platform within 65 $\mathrm{s}$, it was removed from the water and located on the platform for a $30 \mathrm{~s}$ pause. Rats were dried and kept warm immediately after each test, then they were returned to their home cage. Time in which the rat reached the platform was recorded and was presented as mean \pm standard deviation (SD).

\section{Western blotting analysis}

Elevated content of A $\beta$ and APP proteins are important hallmarks of AD and GFAP is a main biomarker of neural activation and damages that were assessed by using specific antibody in NBML rats and AD rats that received THDOC. According to a standard protocol (22), the proteins have been transferred onto PVDF membrane under $140 \mathrm{~V}$ for $1.5-2 \mathrm{~h}$ in the transfer buffer after SDS-PAGE. Membrane was probed with the primary and secondary specific antibodies after blocking, and was washed four times in TBST (50 mM Tris, $\mathrm{pH} 7.5,150 \mathrm{mM} \mathrm{NaCl}, 0.05 \%$ Tween 20). Bands containing specific proteins were visualized by ECL detection kit according to the manual. Anti $\beta$-actin $(1: 1,000)$ (Cell Signaling Technology) was used as a housekeeping protein to control protein concentration.

\section{Histological studies}

For histological analysis rats were sacrificed in deep ketamine and xylazine anesthesia, perfused with $0.3 \%$ sodium sulphide in $0.1 \mathrm{M}$ phosphate buffer and fixed with $4 \%$ formaldehyde. The separated brains were stored in $30 \%$ sucrose for cryoprotection, and sectioned on a freezing microtome at $8 \mu \mathrm{m}$. Tissue sections were stained by hematoxilin-eosin (23) and were studied by light microscope. Abnormalities and tissue damages were detected and confirmed by a pathologist. Tissue sections were also stained with Thioflavin T, a general marker for amyloid deposits staining. For this purpose, tissue sections were incubated with freshly filtered $0.05 \%$ Thioflavin T solution in PBS for $8 \mathrm{~min}$ at room temperature (24). Resulting tissue sections were mounted and studied by fluorescent microscope.

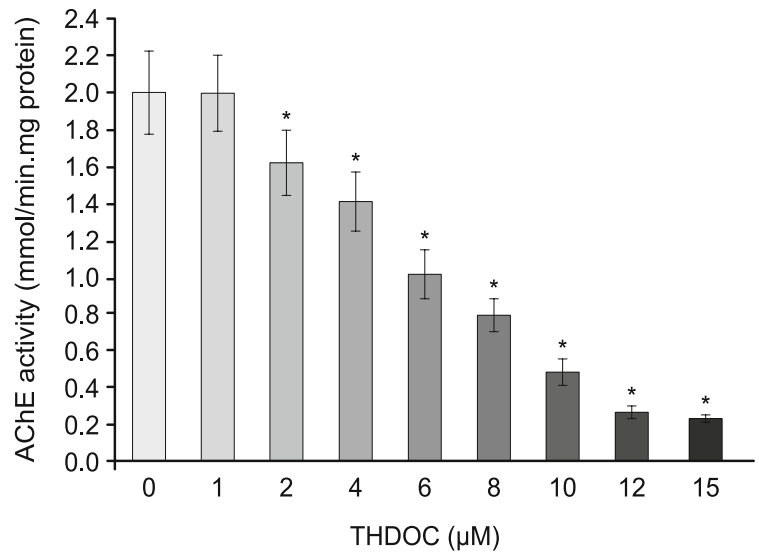

Fig. 1. In vitro assay of $\mathrm{AChE}$ enzyme that was extracted from hippocampal tissue in the presence of THDOC $(0-15 \mu \mathrm{M})$. ACh hydrolyzing activity decreased in the presence of THDOC in a concentration dependent manner. The data are expressed as mean \pm S.E.M. of three independent experiments and asterisk (*) symbol indicates significant changes versus enzymatic activity without neurosteroid.

\section{Statistical analysis}

Data analysis was done by SPSS10 software and in order to identify the difference between experimental groups and control, t-test was performed. Difference in the level $\mathrm{p}<0.05$ was considered significant.

\section{Results}

THDOC binds to the active site gorge and relatively inhibits AChE catalytic activity

A hallmark of AChE is ACh hydrolyzing activity that could be assessed by Ellman method as a sensitive, rapid, and capable to detect very low amounts of catalytic activity (15). According to our previous experiment (15), we used hippocampal tissue homogenate as enzyme source to investigate the possible effects of different concentration of THDOC on catalytic activity of the
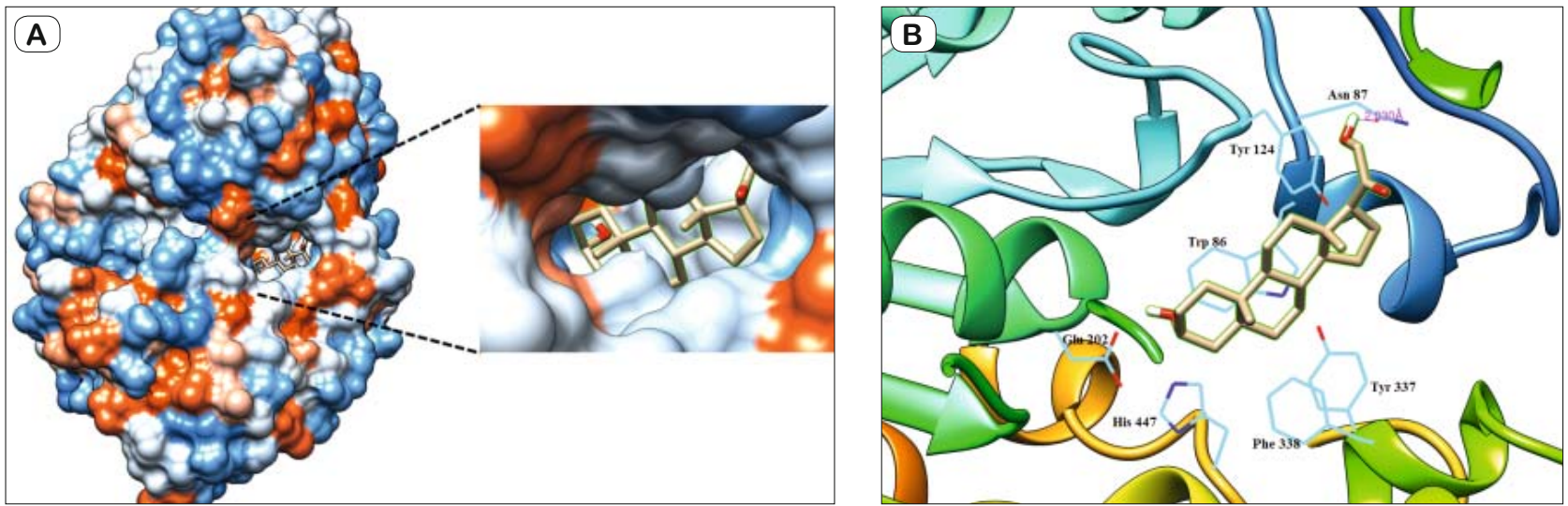

Fig. 2. THDOC molecule in the AChE active site gorge. (A) Surface view of the active site gorge of the crystal structure of AChE with THDOC bound inside in two resolutions. (B) Interaction of THDOC with helix and ribbon related to catalytic site of AChE protein. 

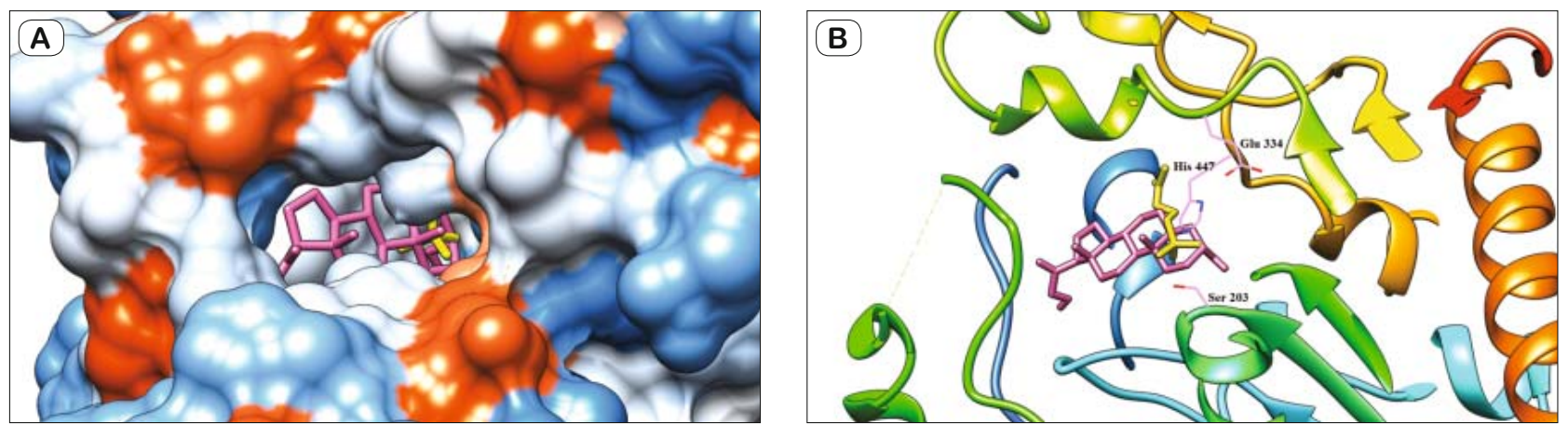

Fig. 3. THDOC and ACh located in the AChE active site. (A) Hydrophobic surface model showed THDOC placed in dipper of the active site gorge exactly such as ACh (substrate) that was suppressed to proper placement in active site. (B) The carton form of one subunit of AChE shows substrate binding residues interact with THDOC (pink molecule) especially with His 447 as a catalytic residue in comparison with ACh was visualized as a yellow molecule in $\mathrm{AChE}$ active site.

AChE. Results showed enzyme activity was reduced in the presence of neurosteroid in a concentration dependent manner (Fig. 1). Maximum inhibition of enzyme activity took place in $15 \mu \mathrm{M}$ of THDOC and IC50 was calculated as $5.68 \mu \mathrm{M}$.

Molecular docking simulation revealed THDOC is capable to bind to the active site gorge of $\mathrm{AChE}$ and the corresponding calculated binding energy is $-9.78 \mathrm{kcal} / \mathrm{mol}$. Crystal structure of human AChE revealed active site gorge consists of catalytic residues (Ser 203, His 447 and Glu 334) that are located at deep of the gorge and some anionic and hydrophobic amino acids that surrounded entrance of the gorge (Tyr 286, Trp 286, Phe 338, Val 294 and Leu 289) known as PAS site $(3,6,25)$. Figure 2 shows the best binding site of THDOC molecule on AChE protein and its position in active site gorge. By considering inhibitory effects of neurosteroid and docking results, THDOC competes with ACh molecule and inhibits substrate binding to catalytic triad, specially
His 447 (3, 25) (Fig. 3). Analyzing the docking results with Discovery studio software revealed THDOC binds to the His 447 by hydrophobic bond it could also bind to Trp 86, Tyr 124, Tyr 337 and Phe 338 by hydrophobic interactions and also binds to Asn 87 by hydrogen bond.

\section{THDOC improved special learning ability in NBM lesioned rats}

Morris water maze task (MWM) is supposed to measure spatial memory, movement control, and cognitive mapping that evaluated hippocampal-dependent learning ability and long-term spatial memory in animal models of cognitive disorders such as Alzheimer disease (21). As expected, control rats quickly learned to swim directly to the platform. NBM lesioned rats that received saline during 25 days reached the platform in significantly more time compared to control rats $(\mathrm{p}<0.05)$ (Fig. 2). Treatment of AD rats with neurosteroid significantly decreased the time delay to reach
(A)

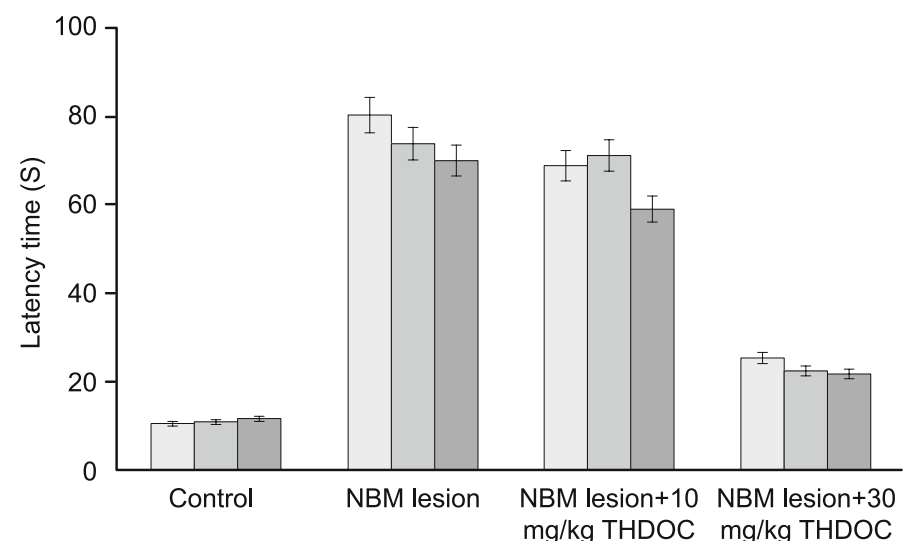

(B)

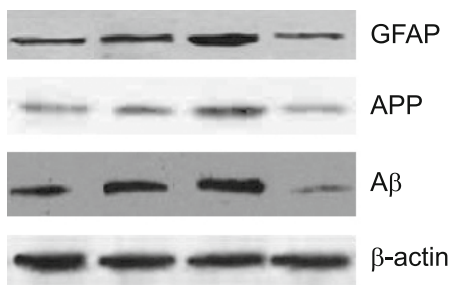

$$
\square 1^{\text {st }} \text { day } \quad \square 2^{\text {nd }} \text { day } \quad \square 3^{\text {rd }} \text { day }
$$

Fig. 4. (A) Spatial learning evaluation by Morris water maze test. Latency of rats in reaching the platform increased in NBM lesioned rats in comparison with control in 3 consecutive test days. $30 \mathrm{mg} / \mathrm{kg}$ of THDOC significantly decreased latency time of AD rats in reach to the platform. Treatment with $10 \mathrm{mg} / \mathrm{kg}$ dose improved spatial navigation in NBM lesioned rats slightly but not significantly. Each data indicates the mean \pm S.E.M. The stares indicate significant differences $(p<0.05)$ according to Duncan's multiple range test. (B) Western blotting analysis reveelad higher expression of GFAP, APP and Aß proteins in AD rats that was reduced by THDOC treatment significantly. 


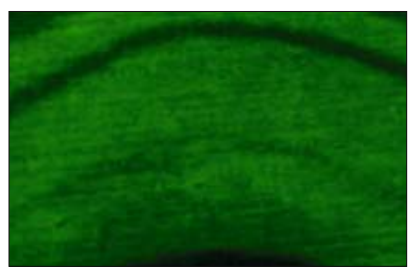

Control

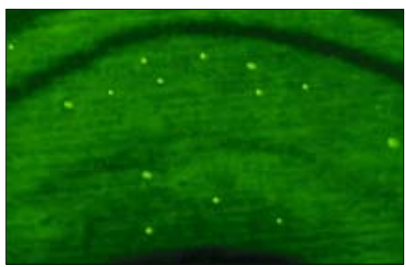

$10 \mathrm{mg} / \mathrm{kg}$ THDOC

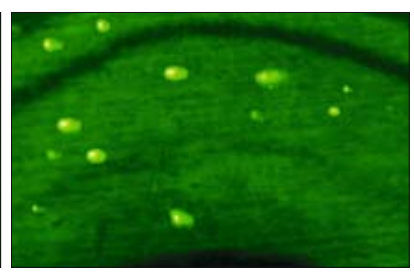

NBM lessioned

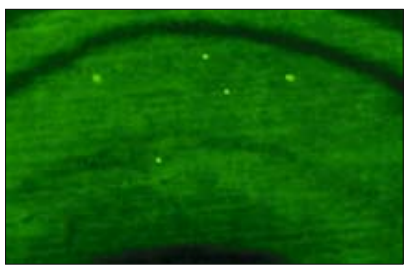

$30 \mathrm{mg} / \mathrm{kg}$ THDOC
Fig. 5. Thioflavin staining of hippocampus tissue sections. Results showed healthy condition in control rats that confirmed by absence of amyloid deposits. Hippocampal sections related to AD rats showed high amounts of deposited plaques. Amyloid deposits amounts and size are reduced in rats that received THDOC.

the platform, especially treatment with high dose of THDOC that reduced latency time more than 3 fold. As is shown in Figure 4, treatment of AD rats with $10 \mathrm{mg} / \mathrm{kg}$ of neurosteroids doesn't have significant improving effect $(p<0.05)$ in same time duration.

THDOC treatment attenuated hydrolytic activity of hippocampal AChE that was induced by NBM lesion

The pathogenesis of AD has been linked to AChE activation that was also accompanied by $\beta$-amyloid deposition $(1,25)$. Therefore to investigate whether NBM lesion and oral administration of neurosteroid supplement affected $\mathrm{AChE}$ activity in rat hippocampus, we evaluated enzyme activity in tissue homogenate related to all groups after experimental duration. In agreement with our previous study (14), results showed AChE activity significantly increased in NBM lesioned rats up to $3012.21 \pm 183.76$ $\mathrm{nmol} / \mathrm{min} . \mathrm{mg}$ protein, while enzyme activity in control hippocampus was $521.36 \pm 82.23 \mathrm{nmol} / \mathrm{min} . \mathrm{mg}$ protein. The rats that administrated by NBM lesion and treated by $10 \mathrm{mg} / \mathrm{kg}$ THDOC during 25 days showed reduced AChE activity (2372.37 \pm 167.23 $\mathrm{nmol} / \mathrm{min} . \mathrm{mg}$ protein). $30 \mathrm{mg} / \mathrm{kg}$ THDOC administration reduced AChE activity in AD rats more than the lower dose and returned ACh hydrolytic activity near to the control, $638.15 \pm 92.12 \mathrm{nmol} /$ min.mg protein.

\section{THDOC improved abnormal expression of GFAP, APP and A}

GFAP is one of the main important biomarkers in neuronal damages that is increased in CNS tissue related to AD patient and models (26). Western blotting analysis confirmed increased expression of GFAP in hippocampus tissue as a result of NBM lesion. GFAP content of hippocampus tissue was reduced in rats that were treated by neurosteroid, especially in rats that daily received $30 \mathrm{mg} / \mathrm{kg}$ dose (Fig. 4). Blotting results also confirmed amyloid precursor protein (APP) level enhanced in hippocampus tissue in $\mathrm{AD}$ rats. Treatment of $\mathrm{AD}$ rats with neurosteroid during 25 days significantly reduced APP expression. Figure 4 revealed $\mathrm{A} \beta$ protein content increased in $\mathrm{AD}$ rats (more than 10 fold) in agreement with other studies (1) but neurosteroid receiving AD rats showed less amount of $A \beta$ in hippocampus tissue. Decrease of hippocampal $A \beta$ content in the presence of neurosteroid is dose dependent but it did not reach to the control. Control rats that received the same dose of neurosteroid in similar time duration did not show significant changes in GFAP, APP and A $\beta$ content of hippocampus (data not showed).

THDOC relatively improved histopathological abnormalities induced by NBM lesion

By considering biological importance of any changes which are found in tissue sections, this study used histological studies as biochemical results confirmation. Thioflavin staining demonstrated a clear abundance of amyloid plaques in the hippocampus tissue section of NBM lesioned rats whereas deposits were absent in control rats (Fig. 5). Deposited plaque numbers were reduced in hippocampus of neurosteroid-supplemented NBML rats compared to $\mathrm{AD}$ rats. In addition to plaque quantity, THDOC treatment reduced plaque size effectively, especially when administrated in high dose (Fig. 5). Hippocampal tissue sections were stained by hematoxylin/eosin method and the results confirmed abnormal appearance in hippocampus tissue related to NBM lesioned group in comparison with control. Tissue appearance revealed extensive degeneration and pyknosis in hippocampus related to $\mathrm{AD}$ rats such as in previous study (14) (Fig. 6). Figure 6 shows condensed apoptotic cells in NBM lesioned hippocampus that are reduced significantly by oral administration of 10 and $30 \mathrm{mg} / \mathrm{kg}$ THDOC (Fig. 6). Presence of the small amounts of necrotic and apoptotic cells in THDOC treated tissue sections confirmed administration of both doses has equal and significant improving effects on $\mathrm{AD}$ rat hippocampus.

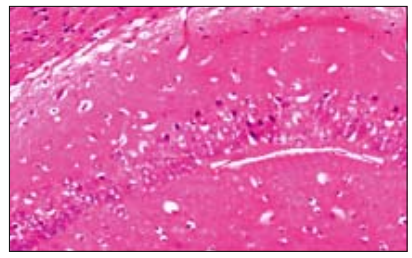

Control

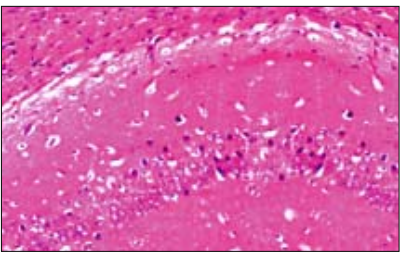

$10 \mathrm{mg} / \mathrm{kg}$ THDOC

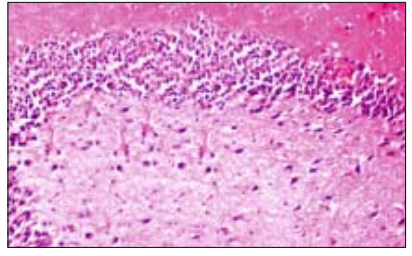

NBM lesion

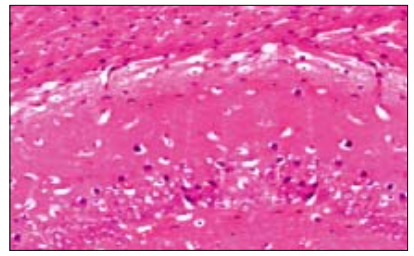

$30 \mathrm{mg} / \mathrm{kg}$ THDOC
Fig. 6. Hematoxylin/eosin staining of hippocampus sections revealed presence of the apoptotic and necrotic cells in NBM lesioned rat hippocampus. Result showed oral administration of THDOC (10 and 30 $\mathrm{mg} / \mathrm{kg}$ ) significantly improved neurodegeneration in NBM lesioned rat hippocampus. 


\section{Discussion}

Neurosteroids are endogenous steroid molecules synthetized in CNS from cholesterol or steroid hormone precursors and have regulatory effects on neuronal functions (9). There are many types of neurosteroids in the brain such as pregnane, androstane and estrogen classes $(9,10)$. The most important neurosteroid in the brain that play essential role in development, function and disease is THDOC (11). THDOC is synthesized from the adrenal hormone deoxycorticosterone as precursor by the action of two enzymes, $5 \alpha$-reductase and $3 \alpha$-hydroxysteroid dehydrogenase in CNS (10). It's a potent positive allosteric modulator of the GAB$\mathrm{A}_{\mathrm{A}}$ receptor, so could be used as sedative, anxiolytic and anticonvulsant (11). Neurosteroids could be used in oral administration for therapeutic purposes due to highly lipophilic properties that let them to easily penetrate through the BBB (12). Improving effects of THDOC and other neurosteroids has been investigated in many neurological disorders (27) but its role in AD has not been investigated previously. Our results revealed THDOC significantly inhibited AChE as a therapeutic target that is hyper-activated in AD patients and related animal models. This study aimed to assess possible effects of THDOC, as a main neurosteroid in CNS, on pathophysiological signs of AD in rat model. We used NBM lesioned rats as prevalent model of $\mathrm{AD}$ that mimics pathophysiological and behavioral signs of AD (19). In this model, local injection of ibotenic acid destroyed ACh producing cells in NBM nucleus of rats (13). We investigated oral administration of THDOC (10 and $30 \mathrm{mg} / \mathrm{kg}$ dose) effects on $\mathrm{AD}$ rats by immunoblotting, biochemical, behavioral and histological assessments. One of the main pathological alterations in $\mathrm{AD}$ is hyper-activation of $\mathrm{AChE}$ enzyme (1) that our results confirmed. THDOC could bind to the active site gorge and inhibit substrate binding to the catalytic site. According to molecular docking results, THDOC could penetrate deep to the gorge and bind to the His 447, Ser 203 and Glu 345 (triad catalytic) and so significantly inhibit hydrolytic activity of $\mathrm{AChE}$ that is assessed by biochemical analysis. Figure 1 shows THDOC significantly inhibited ACh hydrolyzing activity dose dependently and the calculated $\mathrm{IC}_{50}$ was $5.68 \mu \mathrm{M}$. Administration of THDOC to AD rats by effectively reduced AChE activity in hippocampus tissue that was confirmed by in vitro results. In comparison with $\mathrm{AD}$ rats, neurosteroid treatment does not have significant effects in AChE activity and expression level in control rats (data not shown).

In addition to catalytic activity, AChE has some non-catalytic functions by mediating PAS site such as chaperone role in aggregation of the $A \beta$ proteins that lead to plaque formation (3). Our results showed deposited plaques decreased in $\mathrm{AD}$ rats treated by THDOC. Interestingly, results confirmed low dose of neurosteroid causes decreased size of $\mathrm{A} \beta$ plaques and high dose decreased plaque numbers also (Fig. 5). Therefore THDOC inhibits non-catalytic function of enzyme in addition to catalytic activity. Thus our results suggest a new possible role for neurosteroids in $\mathrm{CNS}$ as AChE regulation. Of course decrease in size and number of deposited plaques may be caused by reduced $A \beta$ level that is measured by blotting method, or regulation of other unknown bio- logical process. Previously approved A $\beta$ plaques induce apoptosis in CNS so Alzheimer is considered a neurodegenerative disease (1). Our histological results also revealed degeneration in hippocampal tissue slice in AD rats. Increased expression of GFAP in AD hippocampus also confirmed harsh neuronal damage; GFAP is main biomarker of neuronal damage that is enhanced in neurodegenerative disease (26). AD rats that undergone harsh hippocampal alterations showed neurobehavioral abnormalities also, spatial memory and learning ability decreased significantly as is shown Fig 4. Interestingly THDOC treatment improved tissue appearance in $\mathrm{AD}$ rats and also improved spatial memory that was assessed by MWM test. These and previous results confirmed ACh plays an important role in learning and memory and hippocampus is the main tissue in spatial memory and though ability that undergone harsh alterations in $\operatorname{AD}(15,28)$. Decreased level of the GFAP protein in hippocampus tissue related to $\mathrm{AD}$ rats that were treated by THDOC represents an improvement of the degeneration condition. Previous studies suggested AChE is a multifunctional protein that possibly has a role in gene regulation as a non-catalytic function (5). Simultaneity of AChE inhibition and decreased expression of GFAP, APP, A $\beta$ and other proteins that were not assessed here suggests possible role of $\mathrm{AChE}$ in regulation of gene expression that need further investigation. We used two different doses of treatment and the results approved $30 \mathrm{mg} / \mathrm{kg}$ THDOC improved $\mathrm{AD}$ biochemical and pathophysiological signs more significantly than the lower dose. Regulation of the ACh content in synapses plays an important role in inhibitory and excitatory impulses so our results are in line with previously recognized role of THDOC as GABA activator $(11,28)$. By considering similarity between NBM lesion model and AD in molecular and physiological levels, THDOC could be used as a therapeutic adjuvant in patients suffering from $\mathrm{AD}$ or other related cognitive disorders.

\section{References}

1. Dong S, Duan Y, Hu Y, Zhao Z. Advances in the pathogenesis of Alzheimer's disease: a re-evaluation of amyloid cascadehypothesis. Transl Neurodegener 2012; 1 (1): 1-18.

2. Prasher VP. Review of donepezil, rivastigmine, galantamine and memantine for the treatment of dementia in Alzheimer's disease in adults with Down syndrome: implications for the intellectual disability population. Int J Geriatr Psychiatry 2004; 19 (6): 509-515.

3. García-Ayllón MS, Small DH, Avila J, Sáez-Valero J. Revisiting the Role of Acetylcholinesterase in Alzheimer's Disease: Cross-Talk with Ptau and $\beta$-Amyloid. Front Mol Neurosci 2011; 4: 2.

4. Holmquist M. Alpha/Beta hydrolase fold enzymes: structures, functions and mechanisms. Curr Protein Pept Sci 2000; 1: 209-235.

5. Dong H, Xiang YY, Farchi N, Ju W, Wu Y, Chen L, Wang Y, Hochner B, Yang B, Soreq H, Lu WY. Excessive expression of acetylcholinesterase impairs glutamatergic synaptogenesis in hippocampal neurons. J Neurosci 2004; 24 (41): 8950-8960.

6. Liu Y, Yan B, Winkler DA, Fu J, Zhang A. Competitive Inhibition Mechanism of Acetylcholinesterase without Catalytic Active SiteInteraction: Study on Functionalized $\mathrm{C}_{60}$ Nanoparticles via in Vitro and in Silico Assays. ACS Appl Mater Interfaces 2017; 9 (22): 18626-18638. 
148-154

7. George O, Vallée M, Le Moal M, Mayo W. Neurosteroids and cholinergic systems: implications for sleep and cognitive processes and potential role of age-related changes. Psychopharmacology (Berl). 2006; 186 (3): 402-413.

8. Reddy DS, Estes WA. Clinical Potential of Neurosteroids for CNS Disorders. Trends Pharmacol Sci 2016; 37 (7): 543-561.

9. Tsutsui K. Biosynthesis and Biological Actions of Neurosteroids in Brain Neurons. Zoolog Aci 2001; 18: 1043-1053.

10. Reddy DS. The role of neurosteroids in the pathophysiology and treatment of catamenial epilepsy. Epilepsy Res 2009; 85 (1): 1-30.

11. Reddy DS. Neurosteroids: endogenous role in the human brain and therapeutic potentials. Prog Brain Res 2010; 186: 113-137.

12. Noorbakhsh F, Baker GB, Power C. Allopregnanolone and neuroinflammation: a focus on multiple sclerosis. Front Cell Neurosci 2014; 8: 134.

13. Ackerman HD, Gerhard GS. Bile Acids in Neurodegenerative Disorders. Front Aging Neurosci 2016; 8: 263.

14. Sarnyai Z, Sibille EL, Pavlides C, Fenster RJ, McEwen BS, Toth M. Impaired hippocampal-dependent learning and functional abnormalities in the hippocampus in mice lacking serotonin1 A receptors. Proc Natl Acad Sci USA 2000; 97: 14731-14736

15. Sadeghi L, Yousefi Babadi V, Tanwir F. Improving effects of Echium amoenum aqueous extract on rat model of Alzheimer's disease. J Integr Neurosci 2018; 17 (3-4): 661-669

16. Bradford MM. Rapid and sensitive method for the quantitation of microgram quantities of protein utilizing the principle of protein-dye binding. Anal Biochem 1976; 72: 248-254.

17. Morris GM, Huey R, Lindstrom W, Sanner MF, Belew RK, Goodsell DS et al. AutoDock4 and AutoDockTools4: Automated docking with selective receptor flexibility. J Comput Chem 2009; 30: 2785-2791.

18. Rizvi SMD, Shakil S, Haneef M. A simple click by click protocol to perform docking: AutoDock 4.2 made easy for non-bioinformaticians. EXCLI J 2013; 12: 831-857.
19. Paxinos G, Watson C. The rat brainin stereotaxic coordinates. San Diego: Academic Press; 2005.

20. Irwin RW, Solinsky CM, Brinton RD. Frontiers in therapeutic development of allopregnanolone for Alzheimer's disease and otherneurological disorders. Front Cell Neurosci 2014; 8: 203.

21. Davoodian-Dehkordi, M. Hojjati, M. Yousefi et al. The effect of hydro-alcoholic extract of dried Ficus carica on spatial learning and memory in mice. J Shahrekord Univ Med Sci 2011; 12: 1-7.

22. Sadeghi L, Rizvanov AA, Salafutdinov II, Dabirmanesh B, Sayyah M, Fathollahi Y et al. Hippocampal asymmetry: differences in the left and right hippocampus proteome in the rat model of temporal lobe epilepsy. J Proteomics 2017; 154: 22-29.

23. Dhandapani S, Subramanian VR, Rajagopal S, Ishioka N, Kumazaki T, Tsuru H. Hypolipidemic effects of Cuminum cyminum L. on alloxaninduced diabetic rats. Pharmacol Res 2002; 46: 251-255.

24. Batelli S, Albani D, Rametta R, Polito L, Prato F, Pesaresi M, Negro A, Forloni G. DJ-1 modulates alpha-synuclein aggregation state in a cellular model of oxidative stress: relevance for Parkinson's disease and involvement of HSP70. PLoS One 2008; 3: e1884.

25. Johnson G, Moore SW. The peripheral anionic site of acetylcholinesterase: structure, functions and potential role in rational drug design. Curr Pharm Des 2006; 12 (2): 217-225.

26. Lei J, Gao G, Feng J, Jin Y, Wang C, Mao Q, Jiang J. Glial fibrillary acidic protein as a biomarker in severe traumatic brain injury patients: a prospective cohort study. Crit Care 2015 12; 19: 362.

27. Rahmani B, Ghasemi R, Dargahi L, Ahmadiani A, Haeri A. Neurosteroids; potential underpinning roles in maintaining homeostasis. Gen Comp Endocrinol 2016; 225: 242-250.

28. Hasselmo ME. The Role of Acetylcholine in Learning and Memory. Curr Opin Neurobiol 2006; 16 (6): 710-715.

Received November 18, 2018. Accepted December 20, 2018. 